\title{
Breaking ground in cross-cultural research on the fear of being laughed at (gelotophobia): A multi-national study involving 73 countries
}

RENÉ T. PROYER (University of Zurich, Switzerland), WILLIBALD RUCH (University of Zurich, Switzerland), NUMAN S. ALI (Ibn Rushd Psychiatric Hospital, Iraq), HMOUD S. AL-OLIMAT (University of Jordan, Jordan), TOSHIHIKO AMEMIYA (Kansai University, Japan), TAMIRIE ANDUALEM ADAL (Addis Ababa University, Ethiopia), SADIA AZIZ ANSARI (Institute of Business Management, Pakistan), ŠPELA ARHAR (University of Ljubljana, Slovenia), GIGI ASEM (North South University Dhaka, Bangladesh), NICOLAS BAUDIN (University of Paris X-Nanterre, France), SOUHA BAWAB (American University of Beirut, Lebanon), DORIS BERGEN (Miami University, USA), INGRID BRDAR (University of Rijeka, Croatia), RUTE BRITES (Universidade Autónoma De Lisboa, Portugal), MARINA BRUNNER-SCIARRA (Universidad Peruana Cayetano Heredia, Peru), AMY CARRELL (University of Central Oklahoma, USA), HUGO CARRETERO DIOS (University of Granada, Spain), MEHMET CELIK (Hacettepe University, Turkey), GRAZIA CESCHI (University of Geneva, Switzerland), KAY CHANG (University of Macau, P. R. China), CHEN GUO-HAI (Guangdong University of Foreign Studies, Guangzhou, P. R. China), ALEXANDER CHERYOMUKHIN (Azerbaijan Psychologists Association, Azerbaijan), MARIA P. Y. CHIK (Hong Kong Baptist University, P. R. China), WLADYSLAW CHLOPICKI (Jagiellonian University, Krakow, Poland), JACQUELYN CRANNEY (University of NSW, Sydney, Australia), DONATIEN DAHOUROU (University of Ouagadougou, Burkina Faso), SIBE DOOSJE (University of Utrecht, Netherlands), MARGHERITA DORE (Lancaster University, United Kingdom), NAHWAT EL-AROUSY (Helwan University, Cairo, Egypt), EMILIA FICKOVA (Slovak Academy of Sciences, Slovakia), MARTIN FÜHR (University of Aalborg, Denmark), JOANNE GALLIVAN (Cape Breton University, Canada), HAN GELING (Shanghai University of Science and Technology, Shanghai, China), LYDIA GERMIKOVA (Turkmenistan Association of Psychology/Turkmenistan), MARIJA GIEDRAITYTE (University of Vilniaus, Lithuania), ABE GOH (Oita University, Oita, Japan), REBECA DÍAZ GONZÁLEZ (University of Puerto Rico at Humacao, Puerto Rico), SAI KIN HO (Hong Kong Baptist University, P. R. China), MARTINA HŘEBÍCKKOVÁ (Institute of Psychology, Academy of Science of the Czech Republic, Czech Republic), BELEN JAIME (Argentina), BIRGIT HERTZBERG KAARE (University of Oslo, Norway), SHANMUKH KAMBLE (Karnatak University, India), SHAHE KAZARIAN (American University of Beirut, Lebanon), PAAVO KERKKÄNEN (University of Joensuu, Finland), MIRKA KLEMENTOVÁ (Comenius University, Bratislava, Slovakia), IRINA M. KOBOZEVA (Moscow State Lemonosov University, Russia), SNJEZANA KOVJANIC (University of Zurich, Switzerland), NARASAPPA KUMARASWAMY (University Malaysia Sabah Kotakinabalu, Malaysia), MARTIN LAMPERT (Holy Names University, USA), CHAO-CHIH 
LIAO (National Chiayi University, Taiwan), MANON LEVESQUE (Université Omar Bongo, Gabon), ELENI LOIZOU (University of Cyprus, Cyprus), ROLANDO DÍAZ LOVING (National Autonomous University of Mexico, Mexico), JIM LYTTLE (Penn State Great Valley School of Graduate Professional Studies, USA), VERA C. MACHLINE (Pontifical Catholic University of São Paulo, Brazil), SEAN MCGOLDRICK (Queens University, Belfast, Ireland), MARGARET MCRORIE (Queen's University Belfast, Northern Ireland), LIU MIN (National Education Examination Authority, China), RENÉ MÕTTUS (University of Tartu, Estonia), MARGRET M. MUNYAE (University of Botswana, Botswana), CARMEN ELVIRA NAVIA (National University of Colombia, Colombia), MATHERO NKHALAMBA (University of Malawi, Malawi), PIER PAOLO PEDRINI (University of Lugano, Switzerland), MIRSOLAVA PETKOVA (Trakia University, Bulgaria), TRACEY PLATT (University of Hull, United Kingdom), DIANA-ELENA POPA (University of Galati, Romania), ANNA RADOMSKA (Warsaw University, Poland), TABASSUM RASHID (Aligarh Muslim University, India), DAVID RAWLINGS (University of Melbourne, Australia), VICTOR J. RUBIO (University of Autonoma Madrid, Spain), ANDREA C. SAMSON (University of Fribourg, Switzerland), ORLY SARID (Ben-Gurion University of the $\mathrm{Ne}$ gev, Israel), SORAYA SHAMS (Roudehen University, Iran), SEK SISOKOHM (Royal University of Phnom Penh, Cambodia), JAKOB SMÁRI (University of Iceland, Iceland), IAN SNEDDON (Queen's University, Belfast, Northern Ireland), IRENA SNIKHOVSKA (Zhytomyr State Ivan Franko University, Ukraine), EKATERINA A. STEPHANENKO (Moscow State Lemonosov University, Russia), IEVA STOKENBERGA (University of Latvia, Riga, Latvia), HUGO STUER (Belgium), YOHANA SHERLY ROSALINA TANOTO (Airlangga University, Indonesia), LUIS TAPIA (Villanueva Universidad Del Desarrollo, Chile), JULIA TAYLOR (University of Cincinnati, USA), PASCAL THIBAULT (Université du Québec à Montréal, Canada), AVA THOMPSON (College of the Bahamas, Bahamas), HANNA THÖRN (University of Zurich, Switzerland), HIROSHI TOYOTA (Nara University of Education, Japan), JUDIT UJLAKY (Dániel Berzsenyi University, Szombathely, Hungary), VITANYA VANNO (Srinakharinwirot University, Thailand), JUN WANG (Guangdong University of Technology, Guangdong, China), BETSIE VAN DER WESTHUIZEN (NorthWest University, South Africa), DEEPANI WIJAYATHILAKE (University of Peradeniya, Sri Lanka), PETER S. O. WONG (Universiti Malaysia Terengganu, Malaysia), EDGAR B. WYCOFF (Nicholson School of Communication, University of Central Florida, USA), and EUN JA YEUN (Konkuk University, South Korea)

\section{Abstract}

The current study examines whether the fear of being laughed at (gelotophobia) can be assessed reliably and validly by means of a self-report instrument in different countries of the world. All items of the GELOPH 
(Ruch and Titze 1998; Ruch and Proyer 2008b) were translated to the local language of the collaborator (42 languages in total). In total, 22,610 participants in 93 samples from 73 countries completed the GELOPH. Across all samples the reliability of the 15-item questionnaire was high (mean alpha of .85) and in all samples the scales appeared to be unidimensional. The endorsement rates for the items ranged from 1.31\% through $80.00 \%$ to a single item. Variations in the mean scores of the items were more strongly related to the culture in a country and not to the language in which the data were collected. This was also supported by a multidimensional scaling analysis with standardized mean scores of the items from the GELOPH $\langle 15\rangle$. This analysis identified two dimensions that further helped explaining the data (i.e., insecure vs. intense avoidant-restrictive and low vs. high suspicious tendencies towards the laughter of others). Furthermore, multiple samples derived from one country tended to be (with a few exceptions) highly similar. The study shows that gelotophobia can be assessed reliably by means of a self-report instrument in cross-cultural research. This study enables further studies of the fear of being langhed at with regard to differences in the prevalence and putative causes of gelotophobia in comparisons to different cultures.

Keywords: Cross-cultural comparisons; gelotophobia; humor; laughter; multi-national study.

\section{Introduction}

Laughter is an innate emotional expression in human beings, having a distinct facial and vocal pattern (Ruch and Ekman 2001). Therefore, it is a reasonable assumption that laughing at others will also be a known phenomenon across all cultures and regions of the world. There is empirical data that people get laughed at for a broad variety of reasons. In a recent study, Proyer, Hempelmann, and Ruch (this issue) identified 102 different reasons for being laughed at. By means of a corpus study, they reviewed written records of what actually happened to people when they got laughed at (based on newspaper reports, books, etc.).

Although these reasons were extracted from sources in the German language, it can be assumed that a comparable number of reasons exist in different regions of the world. Thus, it can be predicted that forms of good- and bad-natured laughter exist all over the world. Hence, it is a 
reasonable hypothesis that people who fear being laughed at by others (i.e., gelotophobes) can be found all over the world. So far, there is preliminary data on the existence of gelotophobia in a number of different countries. In a survey Ruch (2002) collected data from 39 countries on all five continents. In total, 116 informants completed a humor survey that also entailed questions relating to the fear of being laughed at. Among others, informants were asked how strong is the fear of being laughed at in the average person in their country, and how much does it affect their behavior? Most of the respondents admitted that the fear of being laughed at does exist in the average person in their culture and that it is fairly strong in affecting how they behave. Interestingly, 17\% felt that laughing at is even more prevalent in their country than laughing with others. Only $5 \%$ of the informants said that it is not at all characteristic in their culture or that it does not exist. Furthermore, there were gender differences among the informants in the appraisal of whether the fear of being laughed at exists in the respective country (higher endorsements by women). Overall, this study provides initial evidence on the global existence of the fear of being laughed at. However, the number of informants from some countries was low and some continents were not well represented.

The main objective of this study is to enable further cross-cultural studies on gelotophobia by showing that the fear of being laughed at can be assessed in a reliable way by means of a self-report instrument in different regions of the world. Moreover, it provides useful information in different cultural contexts (e.g., by showing that different item contents are important in different countries). However, predictions on the existence of gelotophobia in different places of the world are difficult since no empirical data exists. The uncertainty about the global existence of the fear of being laughed at is based on several factors. For example, the diagnosis "gelotophobia" cannot be found in clinical classification systems such as the DSM (Diagnostic Statistic Manual) or the ICD (International Classification of Diseases). Another important point is that the scientific study of the fear of being laughed at has started only recently. Since the concept was first observed among German patients, as was all the work on deriving the concept, defining and measuring it was done in the German speaking countries. Therefore, its validity might be limited to this single cultural background. Thus, it might well be that the fear of being laughed at is only a local phenomenon and not as prevalent in other parts of the world as it is in the German-speaking countries. 
Overall, it is expected that the fear of being laughed at exists to a certain degree in all countries in the world, but it is also expected that countries will differ in their prevalence rates (e.g., determined by cut-off scores in a subjective measure for gelotophobia; see Ruch and Proyer 2008b). However, before prevalence rates can be computed, it has to be shown that the data from a country is stable across different independent samples from that country. Thus, it will be necessary to collect more than one sample from some countries (e.g., large countries like China or the United States). It is expected that there might be regional differences across large countries, but that the general tendencies will be comparable (i.e., a similar expression of mean scores in all items) and that independent samples that are collected in the same country (same town or a near-by region) will be highly similar in their profiles. Furthermore, it is expected that cultural factors contribute to the endorsement of the items in a gelotophobia self-report instrument. Among other criteria, the items of the questionnaire were chosen based on prototypicality ratings for the experiential world of gelotophobes (see Ruch and Proyer 2008b). Thus, the country-specific averaged endorsement to the statements represents the degree to which the item is of relevance in the respective country.

\subsection{Choosing research samples for a multi-national study on gelotophobia}

In the first empirical studies on gelotophobia (Ruch and Proyer 2008a, 2008b) using a large sample of $N=495$ normal controls, no relationship was found between the fear of being laughed at and age, marital status, or the size of town in which the participants lived. Thus, it was decided that none of these demographic variables needed to be individually considered, especially when setting up the conditions for the collection of the samples. Though there were no gender differences in these first studies, we decided to collect data from men and women as gender differences in different countries might occur due to cultural specificities of the respective country. The collaborators had no restrictions regarding the composition of the samples except that all participants had to be 18 years of age or older.

All contributors to the present study were asked to provide a minimum sample of 100 males and 100 females from their country. In most cases researchers from institutions of higher education were asked to 
participate. Thus, most of the samples consisted of university students. Because the gelotophobia measure has a standard deviation of approximately .50 , a sample size of 200 participants yields a standard error of measurement for the mean of approximately .04. Thus, variation in means for different samples from the same population of less than .05 can be attributed to sampling errors and not to genuine differences between the samples. All translations of the questionnaire (the GELOPH) are available for research purposes from the website accompanying the multi-national gelotophobia-project that is hosted by the Zurich-based research group (the website material can be seen at www.psychologie. uzh.ch/perspsy/gelotophobia/) and also upon request from the Zurichbased authors. Table 1 gives an overview on the languages in which the data were collected in the countries represented in the study.

Table 1 shows that 42 different language versions of the GELOPH were used in the present study. Additionally, the table shows that in almost all cases the questionnaire was administered in the local language of the country; if not, the questionnaire was administered either in the teaching language of the respective facility (e.g., English in Saudi Arabia) or in a language that participants spoke fluidly. The contributors were allowed to include country-specific adaptations of the items (e.g., for AUS-, UK- and US-English, or European vs. South-American Spanish etc.).

\subsection{Aims of the present study}

The present study has three main objectives. The first examined whether or not gelotophobia can be assessed in a reliable way across different countries by means of a self-report instrument and whether the data provide useful information on the different countries. Thus, information on the corrected item total correlation for each item of the GELOPH, information on the internal consistency of the questionnaire, and on its factorial structure (loadings of the item on the first factor and comparison of the Eigenvalues across the countries) will be presented.

Second, the study was aimed at providing evidence for the existence of the fear of being laughed at (gelotophobia) in different places of the world as manifested in different thoughts and actions in response to a type of situation; i.e., a comparison of the (mean) item endorsement to the items of the GELOPH. For each item an appreciable number of people should endorse the "slightly agree" and "agree" options; that is, they should 
Table 1. The 42 language versions of the GELOPH used in the present study

\begin{tabular}{|c|c|c|c|}
\hline Language & Language version used in & Language & $\begin{array}{l}\text { Language version } \\
\text { used in }\end{array}$ \\
\hline Afrikaans & South Africa & Japanese & Japan \\
\hline Arabic & Egypt, Iraq, Jordan, Lebanon & Kannada & India \\
\hline Bengali (Bangla) & Bangladesh & Khmer & Cambodia \\
\hline Bulgarian & Bulgaria & Korean & South Korea \\
\hline Chinese & $\begin{array}{l}\text { China, Hong Kong, Macau, } \\
\text { Taiwan }\end{array}$ & Latvian & Latvia \\
\hline Croatian & Croatia & Lithuanian & Lithuania \\
\hline Czech & Czech Republic & Norwegian & Norway \\
\hline Danish & Denmark & Persian & Iran \\
\hline Dutch & Netherlands & Polish & Poland \\
\hline English & $\begin{array}{l}\text { Australia, Bahamas, Botswana, } \\
\text { Canada, England, Ethiopia, } \\
\text { Malawi, Malaysia, Northern } \\
\text { Ireland, Pakistan, Saudi } \\
\text { Arabia, Scotland, South } \\
\text { Africa, Sri Lanka, USA }\end{array}$ & Portuguese & Brazil, Portugal \\
\hline Estonian & Estonia & Romanian & Romania \\
\hline Finnish & Finland & Russian & $\begin{array}{l}\text { Azerbaijan, Russia, } \\
\text { Ukraine }\end{array}$ \\
\hline Flemish & Belgium & Serbian & Serbia \\
\hline French & $\begin{array}{l}\text { Burkina Faso, Canada, } \\
\text { France, Gabon, Switzerland }\end{array}$ & Slovenian & Slovenia \\
\hline German & Austria, Germany, Switzerland & Swedish & Sweden \\
\hline Greek & Cyprus & Setswana & South Africa \\
\hline Hebrew & Israel & Slovakian & Slovakia \\
\hline Hungarian & Hungary & Spanish & $\begin{array}{l}\text { Argentina, Chile, } \\
\text { Columbia, Mexico, } \\
\text { Peru, Puerto Rico, } \\
\text { Spain }\end{array}$ \\
\hline Icelandic & Iceland & Thai & Thailand \\
\hline Indonesian & Indonesia & Turkish & Turkey \\
\hline Italian & Italy, Switzerland & Turkmen & Turkmenistan \\
\hline
\end{tabular}

confirm that the gelotophobic content applies to them. Among gelotophobes these items are endorsed by between $36.3 \%$ and $90.2 \%$ of the people (average item endorsement $=67.4$; Ruch and Proyer 2008b). For a symptom (i.e., item) to be present, one might assume that on average $5 \%$ should endorse the symptom/item (i.e., mark the "slightly agree" or "agree" answer category). Thus, the range of item endorsement across different samples from all over the world provides information on the importance of the respective item content in the given country. The higher 
the item endorsement, the more relevant is the described behavior for the expression of the fear of being laughed at in that country.

Third, we examined how similar independent samples from the same country are (typically using the same language version but also different languages, as is the case for Switzerland), and how different countries may have different results despite the use of the same language version. Multiple samples were collected for different reasons. Countries yielding high scores were supplemented by a second or third sample to see whether these high scores could be replicated. This was already verified for a few samples. Multiple sampling was not attempted for countries where data collection proved difficult. For such cases, neighboring countries with a similar culture were examined for comparable results. Furthermore, more samples were collected for large and highly populated countries and/or multilingual countries. In the present study, data from multiple sites were compared for China, Japan, Switzerland, and the United States. It is assumed that the better the different samples from a country converge, the more valid is the cross-cultural assessment of gelotophobia. In addition to gathering multiple samples within a country, comparisons of different countries were undertaken for the Arabic, English, French, and Spanish language versions. The validity of the cross-cultural comparison will be enhanced when it can be demonstrated that differences emerge among the countries using the same language version, and they are more pronounced than differences between the language versions. A Multidimensional Scaling analysis was performed and aimed at the interpretation of content-related axes of different meaning that existed independently from the general level of gelotophobia.

\section{Method}

\subsection{Participants}

In total, 93 samples from 73 countries entered the study. Across all samples 9,542 males and 12,616 females between 18 (17 years only in the Peruvian sample) and 93 years completed the gelotophobia questionnaire (mean across all samples $=25.31$ years and standard deviation across all samples $=7.07$ ). The study comprised a total of 22,610 participants. Most samples consisted of data from more than 100 males and $100 \mathrm{fe}-$ males. Only the Saudi Arabian sample differed strongly regarding the 
gender distribution. Here, it was difficult to collect data from males because of strict gender segregation rules. Thus, the results from this sample can be interpreted for a female population only. In the Austrian sample, parts of the data were taken out of a larger study in which the gender assignment to the scores could not be provided for anonymity reasons (in the other Austrian sample there were approximately $40 \%$ males). Because there were more than the requested 200 participants (264 in total) involved, we assume that there was a reasonable number of males in this sample as well. For the Swedish sample, no information on the age and marital status of the participants was available (all data were collected with university students).

A few other peculiarities of the samples should be highlighted. First, all participants completed the questionnaire in a paper-pencil test. Only in one of the samples from Taiwan (two in total) were the items read to the participants and then they gave their answers on an especially prepared answer sheet. Second, all participants were at least 18 years of age except for the Peruvian sample in which 93 participants (out of 263) were 17 years old. Third, most of the samples consisted of student samples (the mean age for 54 samples was lower than 25 years). However, there were also samples that were collected among older participants in a nonacademic context. Thus, the mean age varied among the countries with the oldest participants being in Belgium (Flemish speaking part of the country) and Denmark, which both had a mean age above 40 years. Fourth, there were at least 200 participants for most of the samples (thirteen out of 91 were below 200 and they ranged between 177 and 199 in their size). For Bangladesh, Germany, and Spain the sample size was higher than 400 .

\subsection{Instruments}

The GELOPH (Ruch and Titze 1998; in the scoring key by Ruch and Proyer 2008b) is a 15-item questionnaire for the subjective assessment of gelotophobia. All items are positively keyed and they utilize a fourpoint answer scale $(1=$ strongly disagree; $2=$ moderately disagree; $3=$ moderately agree; $4=$ strongly agree). A sample item is "When they laugh in my presence I get suspicious." The items were preceded by instructions, and a set of demographic questions was added (age, gender, and marital status). The GELOPH was used in previous studies and proved to be a valid instrument for the assessment of gelotophobia with 
good psychometric properties (see this issue, and Ruch and Proyer 2008a).

\subsection{Procedure}

The contributors received a standardized procedure for the translation of the questionnaire and the data collection. A detailed description of the concept of gelotophobia (e.g., overview of the current research project, a research paper on gelotophobia, etc.), explanations of the relevant terms, explanations of the whole project and its aims were provided for all contributors. For the translation of the questionnaire, a translation to the local language and an independent back-translation was requested. To ensure that each item had the same meaning in each country, all contributors were invited to discuss their translations and the content of the items with the Zurich-based authors for further information on the meanings. In case there was already a translation to the local language, the contributors were asked to check the applicability of the translation and to suggest adaptations if necessary. Following this approach, biases from inadequate translations were avoided (see Van de Vijver and Leung 1997 for a discussion of possible pitfalls in cross-cultural studies).

After the data collection, all researchers filled in a collaborator's questionnaire providing details on the collection process and on the sample as well as any comments relating to problems or special occurrences while collecting the data. Overall, they did not report any major problems in collecting or processing the data. The collaborators also reported additional information for their sample. For example, the contributors from Australia provided information on the respondents' cultural and linguistic background (e.g., English as a second language, ESL; or nonEnglish-speaking background, NESB). All data were sent either in a standardized data-sheet or as a soft copy to the first authors.

\section{Results}

\subsection{The subjective assessment of gelotophobia across different regions of the world: Evaluation of the usefulness of the GELOPH in cross-cultural settings}

Before the GELOPH can be used in cross-cultural research, information is needed on its psychometric properties in its different language versions. 
For a verification of its usefulness several steps were undertaken. First, information on the lowest and highest corrected item, total correlation for each item, and the median across all samples was compiled. Additionally, the internal consistency (Cronbach alpha-coefficient) across all samples was computed. The median of the alpha coefficients across all samples was .86 indicating high reliability. Next, the factorial structure of the GELOPH was analyzed in all samples separately. The highest, the lowest and the median of the loadings of the first factor from a factor analysis were compared. For the total sample, a one-dimensional solution did fit the data best. The median of the Eigenvalues of the first factor was 5.27; and 1.30 and 1.09 for the second and third factor, respectively. Additionally, the 15 items were intercorrelated across the countries (using the mean for the respective sample, rather than raw scores), and the first unrotated principal component was inspected. Thus, information on the requested one-dimensionality of the items across all samples was available as well. Table 2 shows the statistics for all items of the GELOPH.

Table 2 shows that the median of the corrected item total correlation (CITC) ranged between .35 (item 7) and .58 (item 15). The highest CITCs across all samples were between .61 (item 7 in the Australian sample) and .76 (item 3; Scottish sample). The lowest CITCs were between .02 (one of the Japanese samples; item 7) and .36 (item 15, in the French speaking Swiss sample).

Furthermore, Table 2 shows that the factorial structure in all samples and all different language versions of the GELOPH were highly similar. The scree test typically showed one potent factor, which, on average, explained $36 \%$ of the variance. Except for the Cambodian and the Ukrainian samples, the first Eigenvalue was at least twice the size of the second in all samples. The median of the loadings on the first factor ranged from .43 to .67 across all samples. ${ }^{1}$ The lowest loadings on the first factor ranged between .01 (item 7 in Japan) and .50 (item 12 in Denmark), while the highest loadings ranged between .69 (Denmark, item 7) and .81 (Scotland, item 3; United States/sample from Cincinnati, item 11 and 12).

Strong evidence for the unidimensionality of the 15 items comes from the analysis of the items (i.e., the item mean) across the 93 samples. A principal component analysis based on the intercorrelations among item means yielded a strong first factor (Eigenvalue $=9.08$ ) that explained $60.54 \%$ of the variance. The loadings on the first factor were high and ranged between .66 (item 2) and .88 (item 14). Thus, the items also covaried across samples, not only across individuals. However, two more 
Table 2. Corrected item total correlation and loadings on the first factor for each item of the GELOPH across the 93 samples

Corrected item total correlation (CITC)

1. When they laugh in my presence I get
suspicious.
2. I avoid displaying myself in public
because I fear that people could
become aware of my insecurity and
could make fun of me.

3. When strangers laugh in my presence I often relate this to me personally.

4. It is difficult for me to hold eye contact because I fear to be assessed in a disparaging way.

5. When others make joking remarks about me I feel being paralyzed.

6. I control myself strongly in order not to attract negative attention so I do not make a ridiculous impression.

7. I believe that I make involuntarily a funny impression on others.

8. Although I frequently feel lonely, I have the tendency not to share social activities in order to protect myself from derision.

9. When I have made an embarrassing impression somewhere, I avoid the place thereafter.

Min C

.20

TKM

.69

USA(Ci)

$\mathrm{MdC}$

Min $\mathrm{C}$

Loadings on the first factor

$\begin{array}{lllllllllll}.17 & \text { LTU } & .76 & \text { SCO } & .55 & .20 & \text { LTU } & .81 & \text { SCO } & .63 & .80 \\ .28 & \text { KHM } & .70 & \text { CHE-F } & .54 & .39 & \text { KHM } & .79 & \text { DNK } & .62 & .85 \\ .22 & \text { UKR } & .72 & \text { USA(Ci) } & .55 & .26 & \text { UKR } & .79 & \text { CHE-F } & .63 & .81 \\ .08 & \text { KHM } & .68 & \text { ZFA } & .47 & .14 & \text { KHM } & .75 & \text { SAU } & .55 & .81 \\ .02 & \text { JPN } & .61 & \text { AUS } & .36 & .01 & \text { JPN } & .69 & \text { DNK } & .43 & .71 \\ .23 & \text { HKG } & .74 & \text { MEX } & .53 & .29 & \text { CHE-F } & .80 & \text { USA(Ci) } & .62 & .85 \\ .20 & \text { LKA } & .70 & \text { SCO } & .52 & .35 & \text { LKA } & .76 & \text { USA(Fl) } & .61 & .82\end{array}$




\begin{tabular}{|c|c|c|c|c|c|c|c|c|c|c|c|}
\hline \multirow[t]{2}{*}{ Items of the GELOPH } & \multicolumn{5}{|c|}{ Corrected item total correlation (CITC) } & \multicolumn{6}{|c|}{ Loadings on the first factor } \\
\hline & Min & $\mathrm{C}$ & Max & $\mathrm{C}$ & $\mathrm{MdC}$ & Min & $\mathrm{C}$ & $\operatorname{Max}$ & $\mathrm{C}$ & $\mathrm{MdF}$ & FA-C \\
\hline $\begin{array}{l}\text { 10. If I did not fear making a fool of } \\
\text { myself I would speak much more in } \\
\text { public. }\end{array}$ & .14 & IDN & .68 & BFA & .52 & .14 & IDN & .76 & BFA & .61 & .71 \\
\hline $\begin{array}{l}\text { 11. If someone has teased me in the past } \\
\text { I cannot deal freely with him forever. }\end{array}$ & .26 & BGR & .75 & $\mathrm{USA}(\mathrm{Ci})$ & .50 & .33 & BGR & .81 & $\mathrm{USA}(\mathrm{Ci})$ & .59 & .69 \\
\hline $\begin{array}{l}\text { 12. It takes me very long to recover } \\
\text { from having been laughed at. }\end{array}$ & .35 & KHM & .74 & $\mathrm{USA}(\mathrm{Ci})$ & .57 & .50 & DNK & .81 & $\mathrm{USA}(\mathrm{Ci})$ & .67 & .77 \\
\hline $\begin{array}{l}\text { 13. While dancing I feel uneasy because } \\
\text { I am convinced that those watching } \\
\text { me assess me as being ridiculous. }\end{array}$ & .17 & GAB & .72 & $\mathrm{SCO}$ & .46 & .24 & NOR & .78 & $\mathrm{SCO}$ & .55 & .68 \\
\hline $\begin{array}{l}\text { 14. Especially when I feel relatively } \\
\text { unconcerned, the risk is high for me } \\
\text { to attract negative attention and } \\
\text { appear peculiar to others. }\end{array}$ & .30 & IRN & .67 & USA(Ci) & .51 & .35 & POL & .75 & $\mathrm{USA}(\mathrm{Ok})$ & .60 & .88 \\
\hline $\begin{array}{l}\text { 15. When I have made a fool of myself } \\
\text { in front of others I grow completely } \\
\text { stiff and lose my ability to behave } \\
\text { adequately. }\end{array}$ & .36 & CHE-F & .73 & $\mathrm{USA}(\mathrm{Fl})$ & .57 & .47 & CHE-F & .80 & USA(Ok) & .66 & .82 \\
\hline
\end{tabular}

$\mathrm{C}=$ country; $\mathrm{Min}=$ lowest $\mathrm{Max}=$ highest $\mathrm{MdC}=$ median of CITCs across all samples; $\mathrm{MdF}=$ median of loadings on the first factor across all samples; FA-C = loadings on the first unrotated factor in a factor analysis of the mean scores for all items across all samples; AUS = Australia; $\mathrm{BFA}=$ Burkina Faso; $\mathrm{BGR}=$ Bulgaria; $\mathrm{CHE}=$ Switzerland $(\mathrm{F}=$ French language part $) ; \mathrm{COL}=$ Colombia; $\mathrm{DNK}=\mathrm{Denmark} ; \mathrm{GAB}=\mathrm{Gabon}$; HKG $=$ Hong Kong; IDN = Indonesia; IRN = Iran; JPN = Japan; KHM = Cambodia; LKA = Sri Lanka; LTU = Lithuania; MEX = Mexico; $\mathrm{NOR}=$ Norway; $\mathrm{POL}=$ Poland; $\mathrm{SAU}=$ Saudi Arabia; $\mathrm{SCO}=$ Scotland; TKM $=$ Turkmenistan; $\mathrm{TWN}=$ Taiwan; UKR $=\mathrm{Ukraine} ; \mathrm{USA}=$ United States of America $(\mathrm{Ci}=$ Cincinnati; $\mathrm{Fl}=$ Florida; $\mathrm{Ok}=$ Oklahoma); $\mathrm{ZFA}=$ South Africa. 
Eigenvalues exceeded unity. These two were 1.32 and 1.00, which explained $8.78 \%$ and $6.61 \%$ of the variance, respectively. This indicated that there were some reliable differences among countries that were independent from the general level of gelotophobia.

For a further examination of these differences we computed a multidimensional scaling analysis (MDS; using the ALSCAL-algorithm). In order to eliminate the variance due to the different gelotophobia levels, the 15 mean scores were standardized for each sample separately. The position of the countries in the two-dimensional space between the axes of the configuration is shown in Figure 1.

Figure 1 shows that the countries were organized in a circular structure with a few outlying samples (e.g., Cambodia, Turkmenistan, and

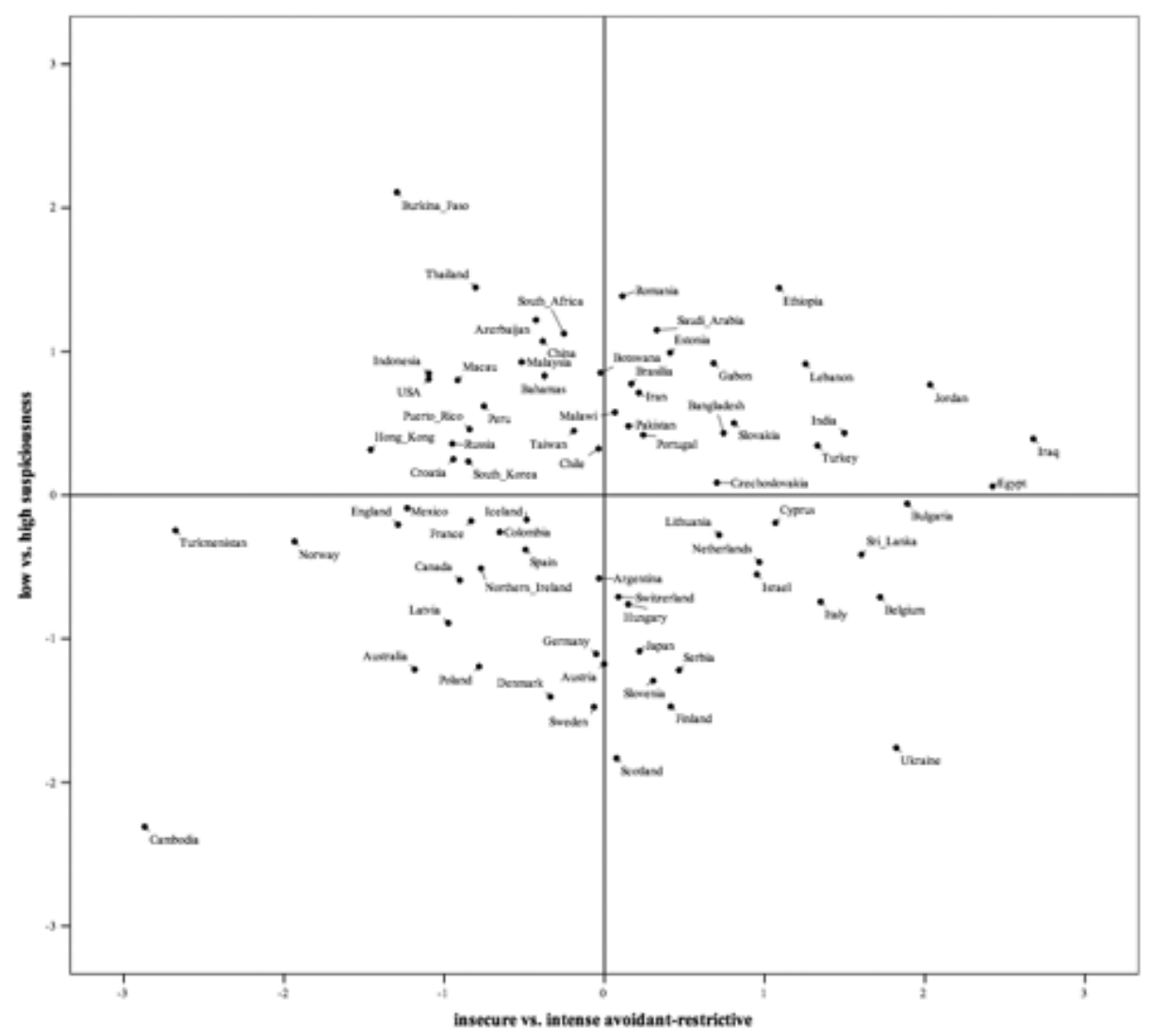

Figure 1. Multidimensional scaling configuration of 73 countries/regions 
Ukraine). Taking the mean scores, factor loadings, and the results from the MDS together (by correlating the factor scores of factor two and three with the country coordinates for the two dimension of the MDS), a meaningful dimensional system emerged. The first dimension represented a specific form of reactions towards the laughter of others and distinguished between insecure (e.g., trying to hide ones experienced insecurity, feeling of being involuntarily funny) and intense avoidant-restrictive reactions towards the laughter of others (e.g., avoiding places where one has been laughed at, feeling uncomfortable if dealing with people from whom one was earlier laughed at, taking a long time for recovering form having been laughed at). Countries with highly insecure reactions were Turkmenistan and Cambodia and those with high intense avoidant-restrictive reactions were Iraq, Egypt, and Jordan. The second dimension referred to low vs. high suspicious tendencies towards the laughter of others (e.g., suspiciousness if others laugh). Countries with highest suspicious tendencies were Burkina Faso, Ethiopia, and Romania and those with the lowest tendencies were found to be Cambodia, Ukraine, and Scotland.

Additionally, the configuration in the MDS showed that countries sharing the same language were clustered near to each other (e.g., Austria and Germany) and geographically neighbored countries also were in similar clusters (e.g., Denmark, Finland, and Sweden). However, there were also exceptions to the rule. For example, countries in which data were collected in English were found in different sections of the configuration and similar clusters were not necessarily related to geographic proximities (e.g., Bangladesh and Slovakia, or Brazil and Malawi). This provided the first evidence that similarities in the outcomes of the questionnaire were not related to a common language used for the data collection or geographic proximities, but presumably more to culture-specific dimensions.

\subsection{Cross-cultural differences in the endorsement of the items from the GELOPH}

In order to allow for a comparison of nation-specific differences in the item-endorsements of the GELOPH, the percentage of answers indicating agreement (i.e., answer categories "moderately agree" and "strongly agree") was computed and compared. As a first overview on crosscultural differences in the item endorsements, the result for item 3, which shows high content validity, was examined first. The country-specific 
average item endorsements ranged from 7.88\% (USA, Cincinnati sample) to $44.39 \%$ (Gabon) with a median of $22.04 \%$. None of the samples was below $5 \%$. The countries were rank-ordered by their item endorsements and it turned out that, on the average, African, Asian, and countries from the Middle East yielded the highest endorsements. We found low endorsements mainly in European, and in North- and South-American countries. However, there were also exceptions. For example, two Chinese samples and Israel were among the countries with low endorsements as well. The differences in the endorsements for all 15 items are shown in Table 3.

Table 3 shows that there was a considerable range in endorsements between the lowest and the highest agreement for the GELOPH-items. The lowest endorsement of a single item was .41\% (item 8, Denmark) and the highest $80.00 \%$ (item 1, Thailand). In 70 samples (out of 93), all items had endorsement rates above 5\%. Items below the 5\% cut-off ranged from .41 to 4.92 with a median of 4.11 . In 19 of the remaining samples, only one item (12 samples) or two items (7 samples) were below 5\%. The exceptions were the samples from Denmark and The Netherlands. Both of them revealed four items with lower endorsement rates than 5\%. After the US sample from Cincinnati, they also had the lowest average item endorsement of all countries (8.53\% for Denmark and $12.17 \%$ for The Netherlands, respectively). The median of the item endorsement ranged between $11.20 \%$ (item 14 ) and $32.91 \%$ (item 6 ).

\subsection{Stability of the item mean scores in gelotophobia in different samples from one country}

The mean profiles from countries with multiple samples were compared (i.e., China, Japan, Switzerland, and the United States). The data from Switzerland is especially interesting since the data were collected in three different languages (all are official languages and spoken in their respective regions; i.e., French, German, and Italian. Additionally, data were collected in French from a bilingual town). Figures $2 \mathrm{a}$ to $2 \mathrm{~d}$ show the mean profiles of the four samples from China, Japan, Switzerland and the six samples from the United States.

Figures $2 \mathrm{a}-2 \mathrm{~d}$ show that the distribution of the profiles was highly similar within the countries. There were outliers for single items (e.g., item 10 for the first Chinese sample) and for whole samples (e.g., the US sample 


\begin{tabular}{|c|c|c|c|c|c|}
\hline GELOPH-items & $\begin{array}{l}\text { Lowest } \\
\text { endorsement }\end{array}$ & $\mathrm{C}$ & $\begin{array}{l}\text { Highest } \\
\text { endorsement }\end{array}$ & $\mathrm{C}$ & MIE \\
\hline 1. When they laugh in my presence I get suspicious. & 8.51 & FIN & 80.00 & THA & 28.54 \\
\hline $\begin{array}{l}\text { 2. I avoid displaying myself in public because I fear that people could become aware of } \\
\text { my insecurity and could make fun of me. }\end{array}$ & 3.28 & NLD & 59.00 & TKM & 19.98 \\
\hline 3. When strangers laugh in my presence I often relate this to me personally. & 4.86 & DNK & 54.40 & THA & 19.19 \\
\hline $\begin{array}{l}\text { 4. It is difficult for me to hold eye contact because I fear to be assessed in a disparaging } \\
\text { way. }\end{array}$ & 3.48 & SRB & 55.50 & KHM & 13.23 \\
\hline 5. When others make joking remarks about me I feel being paralyzed. & 5.97 & SRB & 49.07 & BFA & 18.00 \\
\hline $\begin{array}{l}\text { 6. I control myself strongly in order not to attract negative attention so I do not make a } \\
\text { ridiculous impression. }\end{array}$ & 7.98 & USA(Ci) & 72.65 & IDN & 32.32 \\
\hline 7. I believe that I make involuntarily a funny impression on others. & 4.00 & LKA & 69.42 & IDN & 22.89 \\
\hline $\begin{array}{l}\text { 8. Although I frequently feel lonely, I have the tendency not to share social activities in } \\
\text { order to protect myself from derision. }\end{array}$ & 0.41 & DNK & 71.60 & HKG & 11.80 \\
\hline 9. When I have made an embarrassing impression somewhere, I avoid the place thereafter. & 6.88 & DNK & 64.71 & GAB & 27.58 \\
\hline 10. If I did not fear making a fool of myself I would speak much more in public. & 6.50 & KHM & 73.18 & TWN & 31.12 \\
\hline 11. If someone has teased me in the past I cannot deal freely with him forever. & 3.16 & NOR & 58.59 & EGY & 21.72 \\
\hline 12. It takes me very long to recover from having been laughed at. & 7.18 & USA(Ci) & 55.66 & JPN & 23.49 \\
\hline $\begin{array}{l}\text { 13. While dancing I feel uneasy because I am convinced that those watching me assess me } \\
\text { as being ridiculous. }\end{array}$ & 3.80 & USA(Fl) & 46.94 & MAC & 21.43 \\
\hline $\begin{array}{l}\text { 14. Especially when I feel relatively unconcerned, the risk is high for me to attract } \\
\text { negative attention and appear peculiar to others. }\end{array}$ & 1.31 & NLD & 43.75 & ETH & 10.67 \\
\hline $\begin{array}{l}\text { 15. When I have made a fool of myself in front of others I grow completely stiff and lose } \\
\text { my ability to behave adequately. }\end{array}$ & 2.50 & ROU & 53.77 & EGY & 18.01 \\
\hline
\end{tabular}

$\mathrm{C}=$ country; $\mathrm{MIE}=$ median of item endorsement across all samples; $\mathrm{BFA}=$ Burkina Faso; DNK $=$ Denmark; EGY $=$ Egypt; ETH $=$ Ethiopia; $\mathrm{FIN}=$ Finland; $\mathrm{GAB}=$ Gabon; HKG $=$ Hong Kong; IDN = Indonesia; JPN = Japan; KHM = Cambodia; LKA $=$ Sri Lanka; MAC $=$ Macao; $\mathrm{NLD}=$ Netherlands; NOR = Norway $; \mathrm{ROU}=$ Romania $; \mathrm{SRB}=$ Serbia $;$ THA $=$ Thailand $; \mathrm{TKM}=$ Turkmenistan $; \mathrm{TWN}=\mathrm{Taiwan} ; \mathrm{USA}=$ United States of America $(\mathrm{Ci}=$ Cincinnati; $\mathrm{Fl}=$ Florida $)$. 

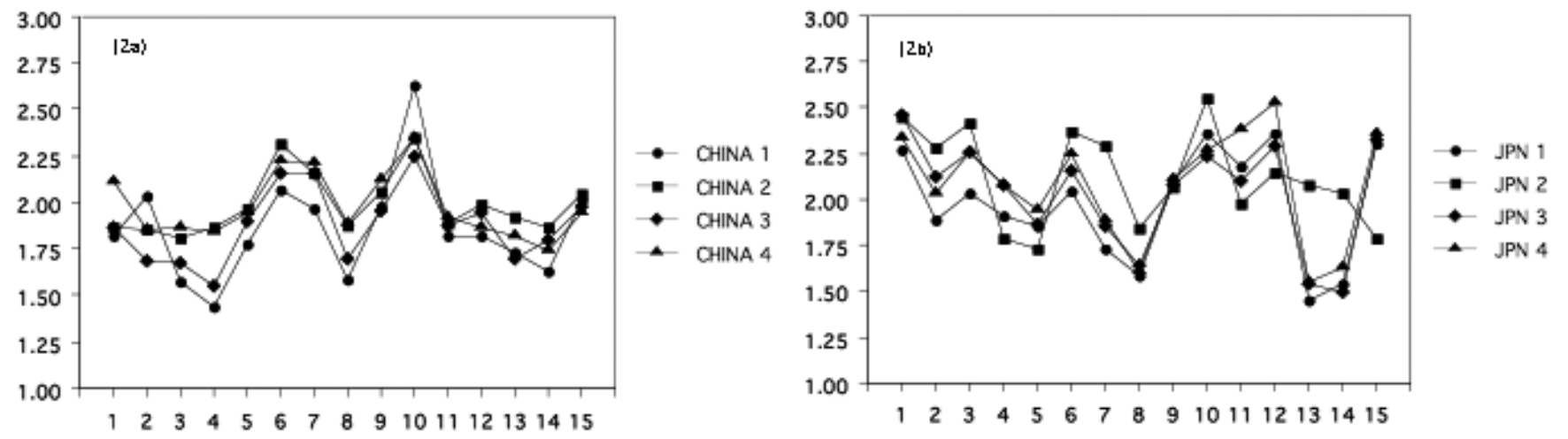

$n$
0
0
7
7
7
0
0
0
0
0
0
0
0
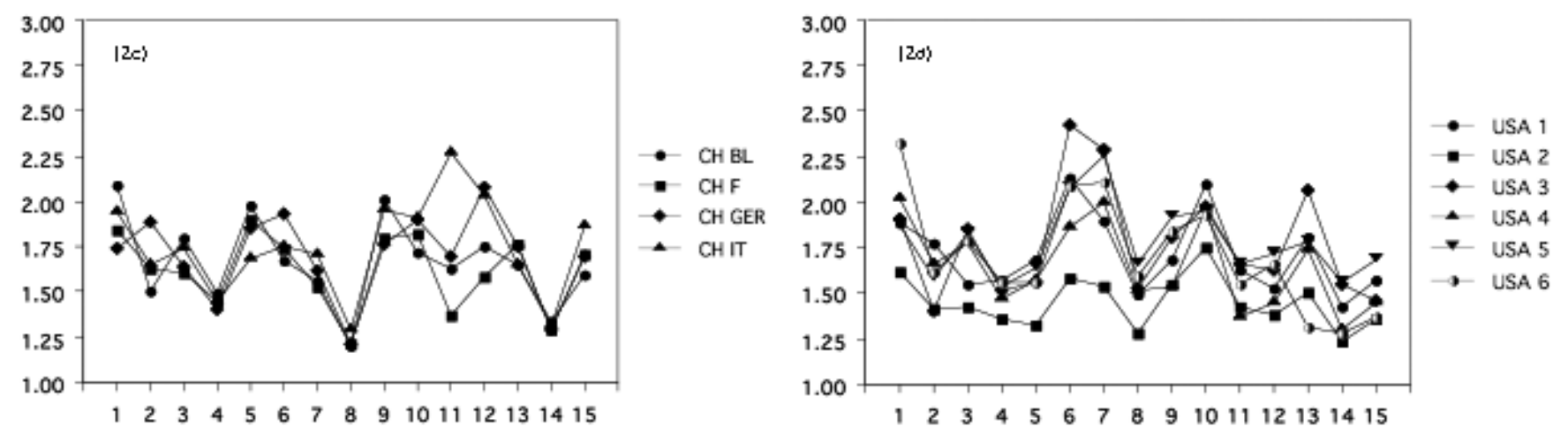

Figure 2. Comparison of the GELOPH-mean scores in the four samples from China (2a), Japan (2b), Switzerland $(2 c)(G E R=G e r m a n$, IT $=I t a-$ lian, $F=$ French; $B L=$ Bilingual, data collected in French), and in the six samples from the United States (2d) 
from Cincinnati; i.e., USA 2). Though the profiles were similar, there were differences among the samples regarding single items. For example, item 11 ("If someone has teased me in the past I cannot deal freely with him forever") in Switzerland. Here, the four samples differed significantly from each other $(F[3,984]=45.77, p<.001)$ with the highest mean for the Italian language part $(M=2.27)$, followed by the German ( $M=1.69)$, the bilingual $(M=1.62)$, and the French language part $(M=1.37)$. All mean scores were significantly different from each other $(p<.05)$ except for the German and the bilingual (French and German) language part. Interestingly, the data from the Italian part of Switzerland was more similar to the data from Italy than to the other parts of Switzerland and the data from the bilingual town was equivalent to that from the French and German language part of Switzerland, but differed from that of the Italian language part $(p<.01)$.

A more promising way of studying the differences among the profiles within one country, rather than the comparison of the mean scores by an ANOVA, might be to use two different parameters. The first one is the correlation across the 15 items (using the mean scores), and the second is the average and highest absolute difference among all samples. The correlations were highest for the Chinese samples (the correlation among the samples was $r=.84$; the average absolute mean difference was $M=.25)$, followed by the US $(r=.64, M=.20)$, Japanese $(r=.62$, $M=.15)$, and Swiss samples $(r=.54, M=.19)$. Overall, the parameters indicated similarity of the samples. That countries did yield homogeneous findings (i.e., higher correlations within a country than between countries) can also be seen from the fact that the average of the 33 correlations within countries was .66 ( $r$ s ranging from .21 to .96), which is high compared to the average of the 120 correlations across the four countries $(M=.39 ; r$ s ranging from -.12 to .83$) .^{2}$

However, Figures $2 \mathrm{a}-2 \mathrm{~d}$ show that on average the profiles were highly similar among samples from one country. Thus, the samples represent a stable estimation of the expression of the relevance of single items (in terms of mean scores) in the respective country. Additionally, the Swiss results indicated that the expression of gelotophobia was more related to the culture in the country than to the language of the questionnaire in which the data were collected. The data collected in three languages (French, German, and Italian) led to similar results. This is also interesting, because among the Swiss samples the lowest overall mean for the comparison of the average absolute differences among all samples was 
found (lowest compared to the Chinese, Japanese, and US sample). Thus, the three different languages were of less importance for the expression of the mean scores than was the shared culture in the country.

A second interesting finding was that, as the Figures $2 a-2 d$ show, there was a considerable difference in the mean scores among the different countries. In general, the profiles from the Asian countries showed higher mean scores than Western countries. Again, this indicated that the expression of gelotophobia (at least the importance of single items) might be related to certain culture-specific norms and values.

\subsection{The relation of the language used in the data collection to the expression of gelotophobia among the different samples}

The influence of the same language used for the data collection in different countries was examined next. Arabic, English, French, and Spanishspeaking countries were compared (at least four different countries for each language). Figure $3 \mathrm{a}-3 \mathrm{~d}$ shows the profiles of the respective countries.

Figures $3 \mathrm{a}-3 \mathrm{~d}$ show that the expression of the mean scores for the items of the GELOPH did not depend on the common language used for the data collection since the profiles among the different countries differed greatly. Clearly, national and cultural differences were more important to explain the differences. Data were collected in Arabic in four countries (Egypt, Iraq, Jordan, and Lebanon). The profiles in Figure 3a indicated that Lebanon had, for most items, the lowest mean scores. For example, in item four there was a high convergence among Egypt, Iraq, and Jordan, but the lowest scores were recorded for Lebanon. It is interesting that there were higher mean scores for Lebanon in item 7 than for the other countries and that the other three countries had almost identical scores in the items 7 and 8 . The highest and lowest mean scores among the four countries differed between .12 (item 1; Lebanon and Iraq) and .60 (item 6; Jordan and Egypt). The average absolute difference among all samples was .22 (ranging from .00 to .60) and the mean of the differences (highest vs. lowest for each item across all four samples) was .41.

The profiles of the French speaking countries (Figure $3 b$ ) revealed even more clearly that the expression of gelotophobia in the items was independent from the common language. Here, the profile from France and 

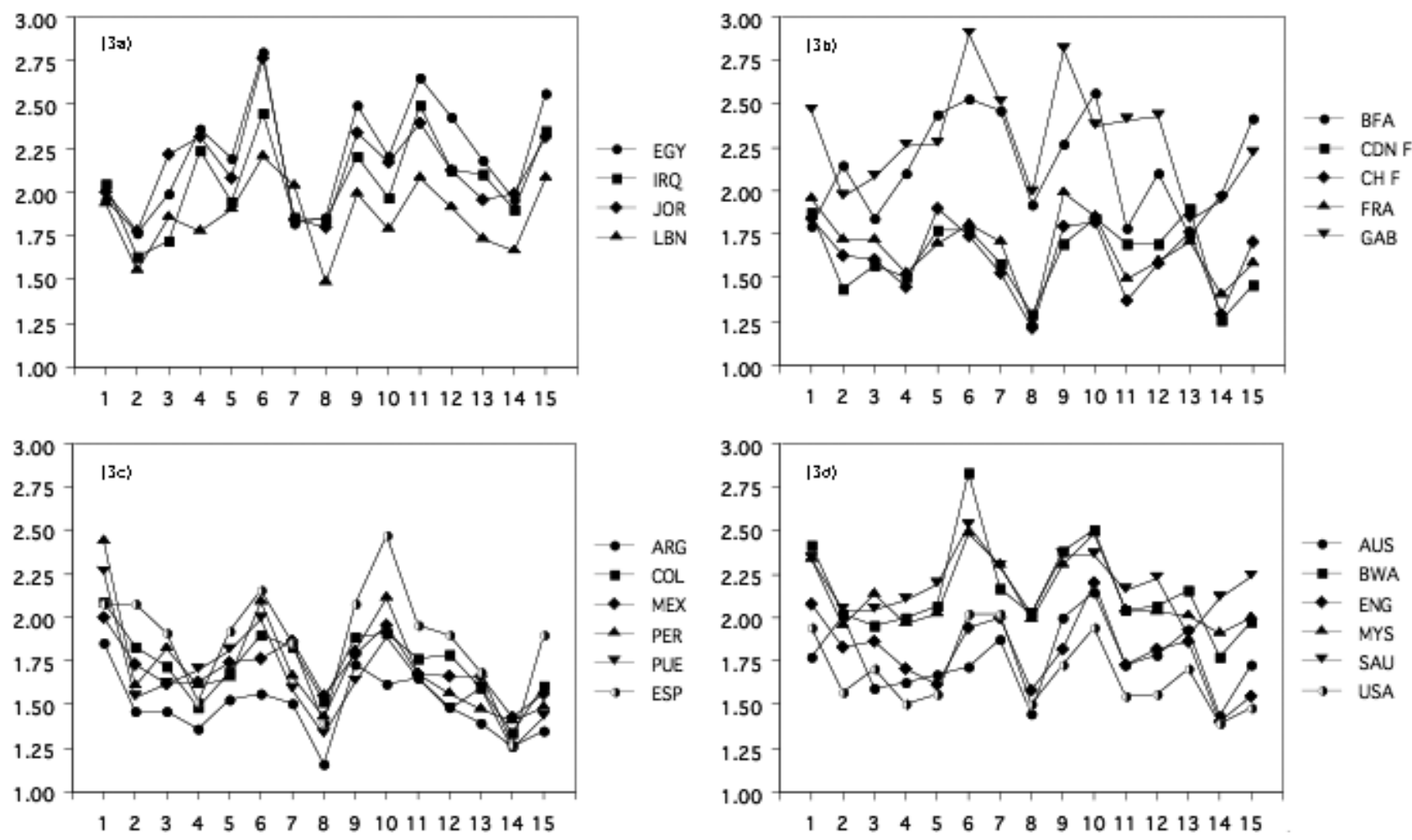

Figure 3. Comparison of the GELOPH-mean scores in samples in which data were collected in Arabic (3a) (Egypt, Iraq, Jordan, Lebanon), French (3b) (Burkina Faso, France, Gabon, French-language part of Switzerland), Spanish (3c) (Argentina, Colombia, Mexico, Peru, Puerto Rico, Spain), and English speaking countries (3d) (Australia, Botswana, England, Malaysia, Saudi Arabia, USA) 
the French speaking parts of Canada and Switzerland was inversely related to the other two countries. However, convergence between the Canadian, French, and Swiss data and the data from Gabon was only found for two items ( 1 and 13 where all five countries converged well). One of the most striking results was that the items 6 and 7 especially seemed to be of higher significance for Burkina Faso and Gabon than it was for the other countries. The average absolute difference among all samples ranged from .01 to 1.16 with a mean of .45 . The mean of the differences between highest and lowest mean among all four samples was .79 and ranged between .16 and 1.16 .

The findings for the Spanish speaking countries were quite similar to the ones reported above (Figure 3c). On the average, the Argentinean sample showed the lowest and the Spanish sample showed the highest means across all items. The highest mean score was found for item 10 in the Spanish sample and this item was of the least importance in Argentina. The best convergence in all items was found for item 14, with highly similar expressions in all samples. The average absolute difference among the samples was .21 (ranging from .00 to .85). The differences between lowest and highest mean differences ranged between .29 (item 13; Argentina and Spain) and .85 (item 10; Argentina and Spain) and had a mean of .45 .

The results for the English speaking samples (Figure 3d) showed that the samples from Australia, England, and the United States were more similar in mean scores of items than the samples from Botswana, Malaysia, and Saudi Arabia. The Western countries were highly similar in the mean scores for item 5, 7, 8, 13, and 14 with almost identical expressions.

However, they differed on items 3, 6 or 12. Overlap in the other English speaking countries was only found in item 2 between Australia and the other non-Western countries and in item 13. Botswana and Malaysia had the highest mean scores and the other countries were highly similar. The average absolute difference among the samples (ranging from .00 to 1.12) was .29. The differences between the highest and lowest mean across all samples ranged between .27 (item 1; US sample and Saudi Arabia) and 1.12 (item 6; Australia and Botswana) with a mean of the differences of .59 .

Overall, the results indicated that the language in which the data were collected did not explain the expression of gelotophobia in the items from the GELOPH; i.e., there were considerable differences in the profiles of countries with the same language. 


\section{Discussion}

The present study was aimed at examining the question of whether or not the fear of being laughed at could be studied in cross-cultural research by means of a 15-item self-report instrument. Overall, the questionnaire, in its translations into 42 different languages, was shown to be a reliable and useful source of information across different nations and cultures.

In all samples the reliability was high (Cronbach alpha between .68 and .92 in all samples; the mean alpha-coefficient was .85). Highest and lowest corrected item total correlations (CITC) were not restricted to single countries but were quite diverse (e.g., the US-sample from Cincinnati shows both, the lowest and highest CITC for an item). This indicates that the measurement of gelotophobia does not work better or worse in a specific country, but that there are specific items that are more or less central to the description of gelotophobia in a specific country. Overall, the reliability and CITCs were high in all samples.

The loadings on the first factor were high in all samples indicating that a single factor solution explained the data best. Thus, in all samples the factorial structure was very similar and very similar to the structure already described in the first empirical studies on gelotophobia (Ruch and Proyer 2008a, 2008b). The fear of being laughed at is best conceptualized as a one-dimensional construct in all countries.

The present study also shows that there are vast differences in the agreement about specific items among the countries. For example, becoming suspicious if others laugh in ones' presence is of high relevance in Thailand $(80 \%$ of the participants agreed or strongly agreed to this item), while the same item endorsement in Finland is much lower (about one tenth; $8.51 \%$ ). Additionally, there is no country that shows the lowest or highest agreement on all items. There are regional differences in the endorsement of specific items. In a future study it might be examined whether the variation in the endorsement of individual items of the GELOPH will also lead to a variation in the expression of the national gelotophobia-scores (i.e., participants in each country that exceed the cut-off points for slight, extreme and pronounced gelotophobia; see Ruch and Proyer 2008b).

It only makes sense to study gelotophobia in different countries if it can be confirmed that the individual samples drawn do, indeed, represent the whole country well. Therefore, multiple samples from China, Japan, Switzerland, and the United States and two samples each from Canada, 
Poland, Russia, Slovakia, and Taiwan were collected and included in the study. The idea was that gelotophobia could be assessed in these countries if the different (independently collected) samples lead to similar expressions in the mean scores of the items. The three main findings from comparison of the items are that: (1) the profiles from different samples from a single country converge well and are similar; (2) the profiles (mean scores) differ between countries (higher and lower expressions and different shapes of profiles); and (3) in Switzerland data were collected in four samples with three different languages (French, German, and Italian) and the profiles were also similar. The latter finding shows that the expression of the mean scores does not depend on the language used for the data collection, but depends more on cultural aspects; for example, one might think of such concepts as collectivism and individualism or independence and interdependence (Dinnel et al. 2002; Hofstede 2001). The first finding is important since it shows that the data collected from different samples of one country converge well.

However, there was also an exception to the rule. In one of the six US samples the mean scores were lower than the other samples. One possible explanation is that the "anonymity condition" in the data collection was not fully warranted in that sample. Some questionnaires were given out individually to friends and neighbors and some of the completed questionnaires were sent in by mail. Thus, it cannot be ruled out that more of the participants than in the other samples may have felt obligated to answer in a socially acceptable manner. Further studies are needed to explain these differences. Overall, the results indicate that it can be assumed that if only a single sample from one country is available, this sample is, nevertheless, indicative of its respective country and representative for data collected under the same conditions.

An analysis of the configuration of the countries/regions disregarding different levels of gelotophobia (i.e., using standardized scores in an analysis of the mean scores of the GELOPH $\langle 15\rangle$ across all countries) led to similar results. A multidimensional scaling analysis helped to identify two dimensions that could be interpreted as insecure vs. intense avoidantrestrictive and low vs. high suspicious tendencies in the reactions towards the laughter of others. The extreme poles of the first dimensions are, for example, Cambodia and Turkmenistan vs. Iraq and Egypt. The second dimension ranges between Burkina Faso and Thailand vs. Cambodia, Scotland, and the Ukraine. The results suggest that geographic proximities and commonly used languages for the data collection are related to 
the position of the countries in the configuration, but do not explain all of the variance fully. Therefore, it is assumed that culture-specific dimensions need to be considered for the further exploration of cross-cultural differences in gelotophobia. This is an objective for further multinational studies on gelotophobia.

The study shows that cross-cultural research on the fear of being laughed at can be conducted by means of self-report data. However, a problem in the use of a subjective measure is that there is empirical evidence that cultural dimensions (e.g., individualism vs. collectivism, uncertainty avoidance etc.) are related to response styles such as acquiescence (see Johnson et al. 2005; Smith 2004). However, as the endorsements to single items vary within the countries, we do not expect strong influences on gelotophobia, but this might be examined in future empirical studies in more detail.

The results of this study enable further cross-cultural explorations of gelotophobia. The clarification of measurement-related question is the basic ground for further studies. In future studies, the way in which prevalence rates of gelotophobia differ among countries and regions will be examined, as well as whether they differ in the expression of extreme cases of gelotophobia. It is expected that there will be a broad variation in the expression of the fear of being laughed at ranging from low to high relevance between different countries. Furthermore, these data will be helpful to identify possible cultural determinants of the fear of being laughed at and possible relations to culture specific dimensions. There are first hypotheses on putative relations between gelotophobia and culture. For example, Davies (this issue) suggests that gelotophobia should be higher in cultures where shame is used as a form of social control and in strongly hierarchical societies. Relating gelotophobia to country-specific dimensions (such as scores on the role of shame in a specific country) can be used to empirically test this hypothesis (e.g., by correlating the country mean scores in gelotophobia with national scores in shame). The data on gelotophobia should also be related to other country-specific data such as overall life-satisfaction scores and economic or geographic data.

\section{Notes}

Correspondence address: r.proyer@psychologie.uzh.ch

* The Czech participation was supported by the research plan AV0Z0250504 of the Institute of Psychology, Academy of Sciences of the Czech Republic and by grant no. 406/ 
07/1561 from the Grant Agency of the Czech Republic. The data collection at the University of Warsaw was supported by the BST grant 1134/49 from 2006. All authors are grateful to the participants for their generous contribution to this study. The authors are grateful to the following persons for their assistance in the data collection or the preparation of the manuscript: Jessica Milner Davis, Tsu Ann Tham, Paul Thomas, Richard Vella (Australia); Daniele Profilo (Bahamas); Jobaer Alam, Rehnuma N. Chowdhury, Khaliquzzaman M. Elias, Tarikul Islam, Umma Jakia, Sajjad Kabir, Khairun Nahar, Nasreen Rahman, Shireen Akhter Ruby, Azmere Sultana Sarah, Abdus Selim and Asefa Zaman (Bangladesh); Bete Dorgam and Yara Kassab (Brazil); Valeri Nikolov (Bulgaria); But Boreth, Khom Chandara, Hoy Chanthoeun, Seng Chhunleng, Ngon Hema, Seng Mang, Khean Munivann, Klot Sambo, On Sodary, Marn Sokhon, Srun Sokkhim, Seng Sophea, Um Sopheap, Phul Sophearith, Kea Sorya, Choeng Sreytom, Ret Thearom, Heng Tola, Ly Vanna, Try Veasna, So Vichet, Vannavuth Sochan Vimean (Cambodia); Liao Jian-hui, Zhou Shaozhou, Chen Wei-qing, Zhang Zhenxiang (P. R. China); Reynel Alexander Chaparro and the undergraduate interns at the Servicio de Atención Psicológica de la Universidad Nacional de Colombia (Colombia); Sameh Salah (Egypt); John S. K. Ng, May M. L. Poon (Hong Kong); Eva Íris Eyjólfsdóttir (Iceland); Seger Handoyo, Endah Mastuti, Budi Setiawan, Rangga P. Wicaksono (Indonesia); A. R. Al-Yassiry (Iraq); Yaacov Doron (Israel); Wafa Adnan (Jordan); Borat Sagdiyev (Kazakhstan); Ivars Austers (Latvia); Stanley Braganza (Macau); William Liew, Noria Raja, Michelle Wong-Lee (Malaysia); Yesica Cienfuegos Mártinez (Mexico); Marie-José van den Berg, Miriam van den Berg, Nina Bolwerk, Geerte de Boois, Jorinde van der Heijden, Marjolein Jochemsen, Hester van Liempt and Josine Peet (The Netherlands); Ian Sneddon (Northern Ireland); Felipe Alva (Peru); Dorota Brzozowska, Joanna Chłopicka, Michał Garcarz, Dariusz Kałuża, Katarzyna Korzec, Marek Kuniak, Maja Lubańska, Andrzej Pawelec, Joanna Sobiecka, Barbara Śpiewak, Anna Wyrwa (Poland); Rodica Apostolatu, Gabriela Scribnic (Romania); Stephanenko Nataliya Alexeevna, Karacheva Ekaterina Anatoliyevna, E. Delikishkina, R. Idrisov, Alyona Ivanova, V. Ponomareva, Lebedev Kirill Sergeevich, (Russia); Mile Kovjanic, Milica Kovjanic (Serbia); Tomáš Chodúr, Hana Luciaková, Martina Magulová, Róbert Nagy (Slovakia); Philipp Drack, Stephanie Estoppey, Julien Flückiger, Noah Savary, Bénédicte Wildhaber (Switzerland); Jennet Germikova, Fewziya Mirzina (Turkmenistan); Jon Acker, Sergio Alatorre, David Annible, Ariana Charles, Heather Gaulden, Chenique Jackson, W. H. A. Johnson, Christina Merlos, Caren Oyor, Lawrence Sherman, Christal Ternate, Alex Thomas, Kaijah Thompson, Victoria Thompson, Rebecca Wagner, Jonathan Wells, and Lauren White (USA).

1. We found negative CITCs in the samples of Burkina Faso and Hong Kong. Item 7 was accountable for both of them and it seems that this was related to an extraordinarily high prevalence rate in these samples. While the median endorsement of this item across all samples was $22.93 \%, 60.00 \%$ and $51.18 \%$ of the participants from Hong Kong and Burkina Faso, respectively endorsed this item (i.e., marked the "agree" or "strongly agree"). This might be related to the fact, that in both samples the translation was based on an older version of the questionnaire, in which the application that people are involuntarily funny was not yet underscored. It cannot be ruled out that participants may have misinterpreted the item ("I believe that I make a funny impression on others"), taking it in the sense of voluntarily making a comic impression on others, and thus, perhaps, leading to higher endorsement rates. Likewise, there was a negative factor loading for this item in the Hong Kong sample.

2. Two samples each were collected in Canada, Colombia, Poland, Russia, Slovakia, and Taiwan. The five within-country correlations averaged at .78 ( $r$ s from .64 to .92 ). The 
average of the 55 correlations across the countries was much lower $(r=.58, r$ s from .20 to .85$)$.

\section{References}

Dinnel, Dale L., Ronald A. Kleinknecht, and Junko Tanaka-Matsumi

2002 A cross cultural comparison of social phobia symptoms. Journal of Psychopathology and Behavioral Assessment 24 (2), 75-84.

Hofstede, Geert

2001 Culture's Consequences: Comparing Values, Behaviors, Institutions, and Organizations across Nations. Thousand Oaks, CA: Sage.

Johnson, Timothy, Patrick Kulesa, Young Ik Cho, and Sharon Shavitt

2005 The relation between culture and response styles: Evidence from 19 countries. Journal of Cross-Cultural Psychology 36 (2), 264-277.

Ruch, Willibald

2002 State of the art in humour research. (Presidential address). 21st International Humor Conference and the 14th Conference of the International Society for Humor Studies, Forli.

Ruch, Willibald and Michael Titze

1998 GELOPH $\langle 46\rangle$. Unpublished questionnaire, Department of Psychology, University of Düsseldorf, Germany.

Ruch, Willibald and Paul Ekman

2001 The expressive pattern of laughter. In Kaszniak, A. (ed.), Emotion, Qualia and Consciousness. Tokyo: World Scientific, 426-443.

Ruch, Willibald and René T. Proyer

2008a The fear of being laughed at: Individual and group differences in Gelotophobia. Humor: International Journal of Humor Research 21 (1), 47-67.

2008b Who is gelotophobic? Assessment criteria for the fear of being laughed at. Swiss Journal of Psychology 67 (1), 19-27.

Smith, Peter B.

2004 Acquiescent response bias as an aspect of cultural communication style. Journal of Cross-Cultural Psychology 35 (1), 50-61.

Van de Vijver, Fons J. R., and Kwok Leung

1997 Methods and Data Analysis of Cross Cultural Research. Thousand Oaks, CA: Sage. 